\title{
Tracking time with song and count: Different Weber functions for musicians and nonmusicians
}

\author{
SiMON GRONDIN \\ Université Laval, Québec, Québec, Canada \\ AND \\ Peter R. Killeen \\ Arizona State University, Tempe, Arizona
}

\begin{abstract}
Participants reproduced target intervals of 6-24 sec with finger taps, with target intervals presented before each of 30 reproductions. During the presentation of the target and reproduction, nonmusician participants were asked to count, sing, or do nothing. In another part of the experiment, musicians were asked to count or sing. Segmentation, either by song or count, minimized deviations from targets and reduced the coefficient of variation $(\mathrm{CV})$ to one third of the nonmusicians' $\mathrm{CVs}$ in the no-segmentation condition. The mean deviation from target was significantly lower for musicians than for nonmusicians, as were the musicians' CVs (half the value of the nonmusicians). Most importantly, although the $\mathrm{CV}$ of nonmusicians was invariant over the range of intervals conforming to a strict scalar property, it decreased significantly for musicians as target intervals increased.
\end{abstract}

If you are asked to keep track of time for a long period - say, $15 \mathrm{sec}$ - it is very likely that you will adopt some strategy in order to segment the target interval into subintervals. You may exploit biorhythms, by counting or estimating breaths or paces while walking, or you may count rhythmic movements, such as tapping your foot or counting numbers-“"One thousand-one, one-thousandtwo"- or steamboats or Mississippis, if you count in English. You are less likely to consider singing. The present study provides an empirical test of the relative efficiency of two mediators, counting and singing, for keeping track of time. We studied this question in conditions in which participants reproduced long intervals. Such timing required the perception of a target interval, its encoding, and finally its reproduction. We interpret the results in the light of contemporary models of time perception.

There are several propositions in the time perception and timing literature regarding the nature of hypothetical mechanisms for processing temporal information, generically referred to as an internal clock. The contemporary model of the internal clock cited most often comprises a pacemaker emitting pulses that are accumulated by a counter (Killeen \& Weiss, 1987; McAuley \& Jones, 2003; Rammsayer \& Ulrich, 2001). The accumulation of pulses in the counter is assumed to be under the control of a switch mechanism that is affected by the amount of attention allocated to time (Casini \& Macar, 1997; Macar, Grondin, \& Casini, 1994). Finally, judgments are based on the comparison of these accumulations with a criterion that had been established by a history of training.
The pacemaker-counter model currently most popular, scalar expectancy theory (SET; Gibbon, 1991, 1992; Gibbon, Church, \& Meck, 1984; Lejeune \& Wearden, 2006), recognizes that the standard deviation $(S D)$ of estimates typically increases as a linear function of the magnitude of the duration to be judged, a property known in psychophysics as Weber's law and in SET as the scalar property of timing. According to Weber's law, the Weber fraction $(w)$ is invariant over the magnitude of the stimuli discriminated. This fraction is typically measured as the coefficient of variation $(\mathrm{CV}=\sigma / t)$, where $\sigma$ is the $S D$ of estimates and $t$ is the interval being judged or produced.

A well-known deviation from this simplest version of Weber's law is an increase of the Weber fraction at small magnitudes. This increase is well captured by the generalized Weber law (Grondin, 2001), which adds a threshold variance, $k^{2}$, to the interval estimates: $\mathrm{CV}=\left(w^{2}+k^{2} / t^{2}\right)^{1 / 2}$. For moderate and large values of $t$, this reduces to the simple Weber's law: $\mathrm{CV}=\sigma / t=w$. Recent data from the animal literature (Bizo, Chu, Sanabria, \& Killeen, 2006) exhibit a clear violation of the scalar property at the other end of the range as well: In two experiments involving long intervals, using both categorization and production methods, the Weber fraction was found to be much larger at $32 \mathrm{sec}$ than at $8 \mathrm{sec}$. Getty (1975) provided one of the most often cited examples of the constancy of the ratio for durations ranging from 0.2 to $2.0 \mathrm{sec}$, but Getty's data also show a substantial increase of the CV for durations above $2 \mathrm{sec}$. Fraisse (1978) summarized numerous studies in human timing that reported increases of the $\mathrm{CV}$ for longer intervals. 
Segmenting time reduces overall variance and seems to prevent increases in the Weber fraction at long durations (Fetterman \& Killeen, 1990; Hinton, Harrington, Binder, Durgerian, \& Rao, 2004; Petrusic, 1984, 2002). Explicit counting (e.g., the quartet of syllables in one-thousandone, etc.) improves performance for intervals longer than $1.2 \mathrm{sec}$ (Grondin, Meilleur-Wells, \& Lachance, 1999; Grondin, Ouellet, \& Roussel, 2004). Below this threshold, the variance added by imperfectly resonant biorhythms and the constant variance added by engagement and disengagement of that mechanism set a floor of $k^{2}$ on the variance of estimates. As $t$ decreases below $1 \mathrm{sec}$, pure timing becomes more accurate than count-mediated timing. Hinton and Rao (2004) reported that for intervals ranging from 8 to $24 \mathrm{sec}$, timing without segmentation conformed to Weber's law (constancy of the CVs of reproductions), but when counting was permitted, the CVs decreased systematically with increasing target durations.

Despite the obvious benefits of segmenting temporal information, the research on this topic rarely covers a large range of durations, is often restricted to counting numbers, addresses only the capacity to make estimates remaining close to real time, and neglects the potential efficacy of song. Singing is a segmentation technique of interest because, in addition to their ability to judge slight tempo variations in a musical excerpt (Grondin \& Laforest, 2004), people can produce stable tempos when asked to recover songs from memory (Levitin \& Cook, 1996) or when imagining or producing songs or nonvocal music (Collier \& Collier, 1994; Halpern, 1988). On the other hand, singing requires greater breath management, possibly entailing greater energy than does counting. This distraction might entail better performance with counting than with singing. ${ }^{1}$

Because the ability to keep a regular pace while singing, and perhaps even while counting, might depend on musical training, a second part of the study involved the participation of musicians. However, it is also known that amateur singers from the general population can remain in time with a well-known tune almost as well as professional singers can (Dalla Bella, Giguère, \& Peretz, 2007). Therefore, any superiority of musicians over nonmusicians for keeping track of time with a singing or a counting strategy may be small or nonexistent.

\section{METHOD}

\section{Participants}

Twelve 21- to 36-year-old ( $M=26.0)$ Laval University student volunteers, 8 females and 4 males, participated in the 12 sessions of the experiment. They received $\$ 60$ Canadian for their participation. None of these subjects were musicians, but they all reported knowing "Au clair de la lune."

Eight 20- to 47-year-old ( $M=29.8)$ musicians (flute, violin, guitar, and, mainly, piano), 2 males and 6 females, also participated in the experiment, but only in the singing and counting portions. ${ }^{2}$ They had 11 to 23 years of musical training $(M=16.6)$. They received $\$ 40$ Canadian for their participation; all reported familiarity with "Au clair de la lune."

\section{Material and Stimuli}

An Pentium IV-based IBM microcomputer running E-Prime software controlled the stimulus presentations. The participants used the space bar of the keyboard to produce intervals. The 20-msec and $44-\mathrm{Hz}$ sounds were generated with a Sound Blaster Audigy 2 sound card and were delivered simultaneously through four Logitech Z-640 speakers located about $1.1 \mathrm{~m}$ apart, with the participant about $1.1 \mathrm{~m}$ in front of the middle speakers.

\section{Procedure}

Nonmusicians experienced 12 experimental conditions: 4 durations $(6,12,18$, and $24 \mathrm{sec}) \times 3$ segmentation conditions (count, sing, no segmentation). For each participant, there were 12 sessions, 1 per experimental condition, lasting about $15-35 \mathrm{~min}$. There was at least a $1-\mathrm{h}$ break between sessions and at least 1 day when the participant undertook sessions in a new segmentation (counting vs. singing) condition. The participants completed 4 sessions ( 1 per duration) with one segmentation condition before starting a new segmentation condition. The order of the duration conditions was randomized according to a Latin square, and for each of the four cycles of the Latin square, there were three orders of segmentation conditions.

The musicians experienced eight experimental conditions: $4 \mathrm{du}-$ rations $(6,12,18$, and $24 \mathrm{sec}) \times 2$ segmentation conditions (count and sing). For each participant, there were eight sessions, one per experimental condition, lasting about 15-35 $\mathrm{min}$. There was at least a 1-h break between sessions, and at least 1 day when the participant undertook sessions in a new segmentation condition. The participant completed four sessions (one per duration) with one segmentation condition before starting a new segmentation condition. The order of the duration conditions was randomized according to a Latin square, and for each of the four cycles of the Latin square, there were two orders of segmentation conditions. Four participants counted first and sang second, and 4 sang first and counted second.

During each session, the participant heard two consecutive sounds marking the target interval to be produced and was asked to reproduce the interval with two finger taps on the space bar. After the reproduction, when ready, the participant initiated the next trial. This presentation-reproduction procedure was repeated 29 additional times. In the counting condition, participants were asked to count at their own pace during both the target presentation and the reproduction phase, looping modulo 10. Participants are asked to use cycles of counts from 1 to 10 in order to avoid the irregularity in the number of syllables above 10 in their native language, French. ${ }^{3}$

In the singing condition, they were asked to sing "Au clair de la lune" in their own manner and at their preferred pace. This song was used because of the general familiarity that many of the Québec participants would have had with it since childhood. The participants were free to count or sing aloud or silently, but were asked to maintain the same strategy throughout the experiment.

In the no-segmentation condition, the participants were asked to not count, sing, or adopt cyclic activities (e,g, moving neck, tapping foot, breathing, imagining a hand ticking on a clock). They reported having conformed to our demand, and the data they produced in this condition suggest that their reports were honest.

\section{RESULTS}

The longest and the shortest reproductions at each interval were excluded from the analysis. This reduces variance due to potentially aberrant cases (Wilcox, 1998). As is generally the case in the study of interval production (McAuley, Jones, Holub, Johnston, \& Miller, 2006), the dependent variables of interest are the mean produced interval (MPI) or the mean deviation from the target interval (i.e., the constant error, $\mathrm{CE}$ ) and the $S D$ of the produced intervals or the $\mathrm{CV}(\mathrm{CV}=S D / \mathrm{MPI}) . C E$ and $\mathrm{CV}$ allow direct comparisons of data from different target intervals.

Figure 1 shows the $C E$ in each of the 12 experimental conditions for nonmusicians (left panel) and in each of the 

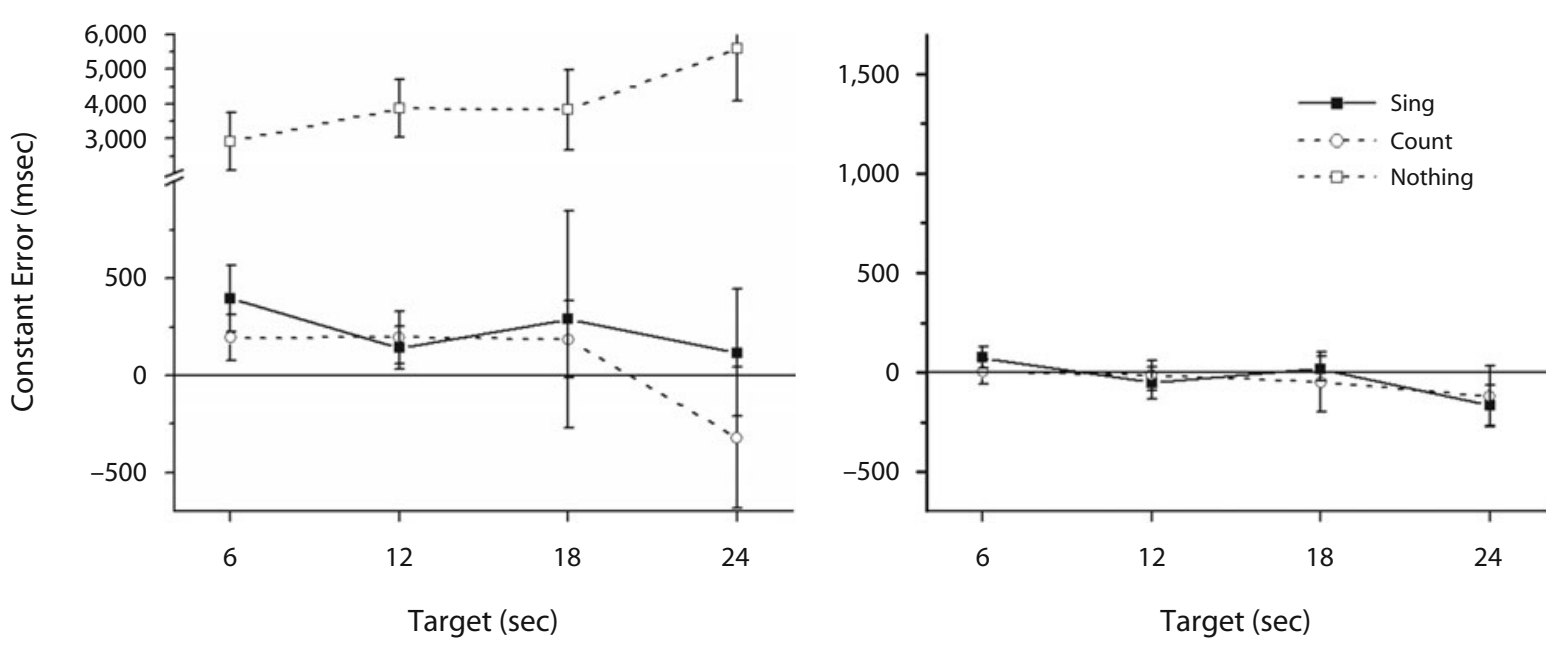

Figure 1. Average constant errors as a function of the target intervals in the singing, counting, and no-segmentation conditions for nonmusicians (left panel) and in the singing and counting conditions for musicians (right panel). Bars represent standard errors.

8 conditions for the musicians (right panel). For nonmusicians, the intervals reproduced in the no-segmentation condition were much longer than those in the singing or counting conditions, and the tendency to produce long intervals in the no-segmentation condition increased as the target intervals got longer, which was not the case for the segmented conditions. The overall mean deviations were 236, 64, and 4,050 msec for the sing, count, and nothing conditions. A 3 (mediators) $\times 4$ (targets) ANOVA with repeated measures on each factor revealed that the target effect was not significant, but both the mediator effect $\left[F(2,22)=21.42, p<.01, \eta_{\mathrm{p}}^{2}=.66\right]$ and the interaction $\left[F(6,66)=3.23, p<.01, \eta_{\mathrm{p}}^{2}=.23\right]$ were significant. Although the $C E$ increased with duration in the no-mediator condition, it decreased in both mediator conditions.

For the group of musicians, a 2 (mediators) $\times 4$ (targets) ANOVA revealed that no effect was significant. Note that both nonmusicians and musicians had their highest mediated $C E$ at $6 \mathrm{sec}(M=296$ and $38 \mathrm{msec}$, respectively) and their lowest at $24 \mathrm{sec}(-102$ and $-144 \mathrm{msec}$, respectively). Overall (singing and counting conditions pooled together), linear analyses conducted on individual data revealed that the intercept was significantly higher for nonmusicians $(M=431 \mathrm{msec})$ than for musicians $(M=$ $93 \mathrm{msec})[t(18)=2.37, p<.05]$.

Figure 2 shows the CVs in each of the 12 experimental conditions for nonmusicians (left panel) and in each of the 8 for the musicians (right panel). For nonmusicians, the $\mathrm{CVs}$ in the no-segmentation condition were much larger than those in the singing or counting conditions, but in all cases, the CVs remained constant over interval. The overall CVs were $.082, .081$, and $.238 \mathrm{msec}$ for the sing, count, and nothing conditions, respectively. A 3 (mediators) $\times 4$ (targets) ANOVA with repeated measures on each factor revealed that the mediator effect was significant $\left[F(2,22)=49.78, p<.01, \eta_{\mathrm{p}}^{2}=.82\right]$, but the target $\left(\eta_{\mathrm{p}}^{2}=.11\right)$ and interaction $\left(\eta_{\mathrm{p}}^{2}=.10\right)$ effects were not.
For musicians, a 2 (mediators) $\times 4$ (targets) ANOVA revealed that there were no mediator $\left(\eta_{\mathrm{p}}^{2}=.004\right)$ and interaction $\left(\eta_{\mathrm{p}}^{2}=.02\right)$ effects. However, the target effect was significant $\left[F(3,21)=4.49, p<.05, \eta_{\mathrm{p}}^{2}=.39\right]$. The CV at $6 \mathrm{sec}(M=.046)$ was significantly higher than the $\mathrm{CV}$ at $24 \sec (M=.030)$, and most importantly, the linear trend was significant $\left[F(1,7)=18.29, p<.01, \eta_{\mathrm{p}}^{2}=.72\right]$, and the quadratic and cubic trends were not. The overall CV (singing and counting for all target conditions) was significantly lower with musicians $(M=.037)$ than with nonmusicians $(M=.081)[t(18)=2.46, p<.05]$.

The number of counts or syllables reached by participants ${ }^{4}$ was divided by the target duration for a measure of rate of mediation. For nonmusicians, a 2 (mediators) $\times$ 4 (targets) ANOVA revealed that there were no target and interaction effects, but the mediator effect was significant $\left[F(1,10)=23.95, p<.01, \eta_{\mathrm{p}}^{2}=.71\right]$, with the number of counts $(M=2.36$ per sec) being much higher than the number of syllables $(M=1.36$ per sec $)$. For musicians, the ANOVA revealed no significant effects $\left(M_{\text {count }}=\right.$ $\left.1.88 ; M_{\text {syllable }}=1.64\right)$.

\section{DISCUSSION}

Extended musical training provided some advantage in minimizing the $C E$ in the singing and counting conditions, inasmuch as that was generally closer to 0 for musicians. But most importantly, the CV was much lower for musicians and decreased as target intervals increased, which was not the case with nonmusicians.

The present study reveals that extensive musical training has a major impact on the ability to minimize variance in performance when keeping track of long time intervals by using a mediator. The average $\mathrm{CV}$ was much lower-half the value-for musicians than for nonmusicians, regardless of whether the mediation was by singing or counting. Perhaps this is not surprising, since keeping time with 

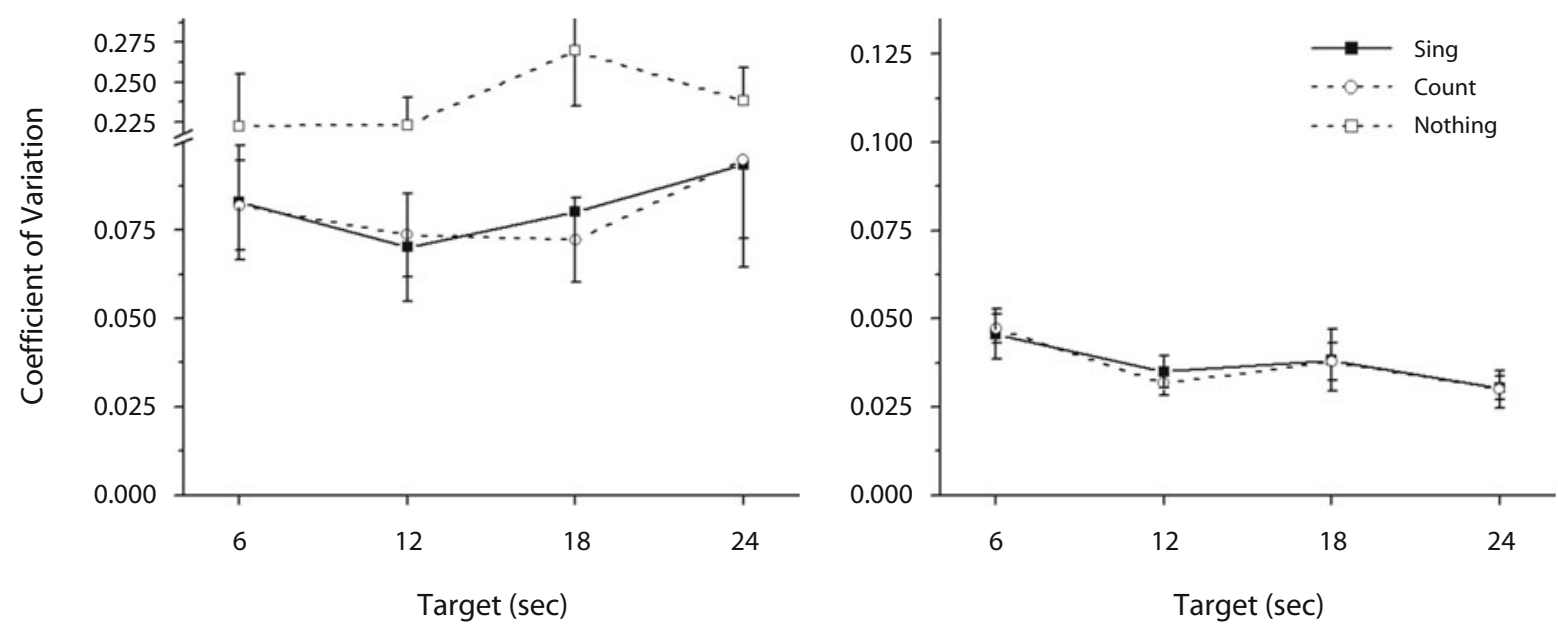

Figure 2. Average $S D$ s as a function of the target intervals in the singing, counting, and no-segmentation conditions for nonmusicians (left panel) and in the singing and counting conditions for musicians (right panel). Bars represent standard errors.

rhythmic movements or even counting explicitly for measuring time is a fundamental skill for musicians; but in light of the demonstration of Dalla Bella et al. (2007) that the temporal variability of occasional singers with a popular song is almost as low as that of professional singers, it suggests that there is more to the good performance of our musicians than just reduction of variance. Our demonstration that extensive musical training can reduce $\mathrm{CV}$ by more than 50\% suggests that other mechanisms, such as reduction of switch variance or of error in going from the presentation to the reproduction phase, may be at work. Other factors, such as the motivation to participate, may also contribute to the observed difference, assuming that musicians, knowing that they were selected on the basis of their musical skills, might have provided more effort in the task. Indeed, the task was very demanding, and we cannot exclude the possibility that the musicians were better prepared for meeting this type of requirement.

Apart from the reduction of the CV, extensive musical training even changes the functional relationship between variability and time. Although SET predicts that the CV should be constant over time (Lejeune \& Wearden, 2006) - a prediction that holds with nonmusicians in both mediator and nonmediator conditions - the CV of musicians decreased with interval length. Hinton and Rao (2004) also reported a violation of the simple Weber's law in a counting condition but reported no specific musical abilities of their subjects. In the present study, the same method led two different groups, those with and without extensive training, to two patterns of results: The CVs of musicians were significantly lower at $24 \mathrm{sec}$ than at $6 \mathrm{sec}$, but with nonmusicians, CVs were, if anything, slightly higher at $24 \mathrm{sec}$ than at $6 \mathrm{sec}$. Kristofferson (1980) showed with a duration discrimination task that extensive training could change the functional relationship between variability and time. For some duration ranges, training led to a reduction of variability to a point where variability per se (not CV) remained constant over interval length. It follows that within those relevant ranges, the $\mathrm{CV}$ would decrease with the interval length. In the present study, extensive musical training did not result in constant variability over time, but variability did grow less than proportionally with time, leading to the decrease in relative variability shown in the right panel of Figure 2.

The overall levels of relative accuracy, as measured by CVs, were much higher in the nonmediated condition than in the mediated one for the nonmusicians. Using mediators, either singing or counting, reduced the CV from about 0.23 to about 0.08 . This finding is consistent with those of Hinton and Rao (2004), who suggested using this $\mathrm{CV}$ difference as a clue to whether the participants were counting in order to time. Note that the nonmediated CVs in the present study $(0.23)$ average half again as large as the constant and nonmediated one ( 0.16$)$ reported by Hinton and Rao for a similar range of intervals $(8-24 \mathrm{sec})$, which was quite similar to the values gotten by Rakitin, Stern, and Malapani (2005) for nonmediated timing of 6 and $17 \mathrm{sec}$ by young (CVs of 0.145 and 0.136 ) and aged (CVs of 0.174 and 0.138 ) participants.

Data with nonmusicians showed that the mediated reproductions of intervals remain much closer to the target, whether they segmented with singing or by counting, than without segmentation. In the nonmediated condition, the reproductions were much too long, by from $3 \mathrm{sec}$ (at $6 \mathrm{sec}$ ) to $5.5 \mathrm{sec}$ (at $24 \mathrm{sec}$ ). This finding shows that keeping track of time without mediators remains very difficult. Not only are CVs three times higher than they are with mediators, but the deviation from target can be as high as half the value of the target. Since the CV is based on this (inflated) mean reproduction, variability in an absolute sense is even worse than the very large CV suggests.

Overall, nonmusicians did not sing and count (overall $M=1.86)$ faster than musicians $(M=1.76)$. However, the nonmusicians sung much faster than they counted. Faster tempo may have generated greater accuracy by taking advantage of Poisson reduction in variance. In spite of this count versus sing difference, nonmusicians did not perform better in the singing than in the counting condi- 
tion. Note that nonmusicians tended to sing faster than musicians, which is consistent with the findings of Dalla Bella et al. (2007), who reported that occasional singers sing at a faster tempo than professional singers do. Finally, that musicians showed no rhythm difference with counts versus syllables is not surprising, since for a musician singing and playing music is constrained by required tempos; counting is a fundamental skill for a musician. Their pace ( 1.76 or $570 \mathrm{msec}$ between counts/syllables) is close to what is sometimes referred to as preferred tempo (McAuley et al., 2006).

We did not conduct a no-segment condition with the musicians, in part because they report finding great difficulty in forbearing to mediate intervals by segmentation strategies of one sort or another. It is not clear whether they would continue to show superior performance in the no-segment condition. It is known that musicians have higher tempo sensitivity than do nonmusicians (Drake \& Botte, 1993; Sheldon, 1994), but we do not know how the groups compare on estimating very long intervals. Indeed, restraining anyone from beating time while estimating long intervals is somewhat unnatural; for musicians, it is reasonable to assume that such restraint could involve inhibition processes that would severely interfere with their performance. As they stand, our results reveal clear superiority in the mode that is natural to most animals, mediated timing.

\section{CONCLUSION}

The present study offers one of the few reports on temporal processing involving a large range of durations, a variety of mediators, and an analysis of both the deviation from target and the variability of temporal reproductions. Not much is known about the variability of longinterval estimates by humans in simpler tasks, and this might be due partly to the fact that, when asked to perform a temporal task involving long intervals, humans and other animals spontaneously subdivide time, if not constrained to do otherwise by experimental procedures (Fetterman, Killeen, \& Hall, 1998). Our study showed that when long intervals were reproduced, deviations from target were greatly reduced by mediation compared to those in a nonmediated condition, where they were substantial; and that singing and counting led to essentially the same average deviations, whether participants were musicians or not. There were, however, significant differences between groups. Not only were the CVs of musicians much lower than those of nonmusicians, but the CVs of nonmusicians remained invariant over interval length, both with and without mediators, whereas the CVs of musicians decreased with interval length. Although both performances were consistent with the generalized Weber's law, only participants who lacked extensive musical training showed conformity with the stricter scalar property. It seems that for this group, performance was dominated by a much larger Weber fraction, $w$, which swamped any potential gains in accuracy that could be realized by moving away from short time intervals and from their burden of threshold variance $k^{2}$.

\section{AUTHOR NOTE}

Portions of this experiment were presented at the 18th Annual Meeting of the Association for Psychological Science (APS) held in New York in May 2006, and other portions at the 2009 APS meeting held in San Francisco. The present research was supported by research grants from the Natural Sciences and Engineering Council of Canada (NSERC) to S.G. and by NSF Grand IBN 0236821 to P.R.K. We thank Nicolas Bisson for his help at various stages of this project and anonymous reviewers for their guidance. Address correspondence to S. Grondin, École de psychologie, Université Laval, Québec, QC, G1K 7P4 Canada (e-mail: simon.grondin@psy.ulaval.ca).

\section{REFERENCES}

Bizo, L. A., Chu, J. Y. M., Sanabria, F., \& Killeen, P. R. (2006). The failure of Weber's law in time perception and production. Behavioural Processes, 71, 201-210.

CASINI, L., \& MACAR, F. (1997). Effects of attention manipulation on judgments of duration and of intensity in the visual modality. Memory \& Cognition, 25, 812-818.

Collier, G. L., \& Collier, J. L. (1994). An exploration of the use of tempo in jazz. Music Perception, 11, 219-242.

Dalla Bella, S., Giguère, J.-F., \& Peretz, I. (2007). Singing proficiency in the general population. Journal of the Acoustical Society of America, 121, 1182-1189.

Drake, C., \& Botte, M.-C. (1993). Tempo sensitivity in auditory sequences: Evidence for a multiple-look model. Perception \& Psychophysics, 54, 277-286.

Fetterman, J. G., \& Killeen, P. R. (1990). A componential analysis of pacemaker-counter timing systems. Journal of Experimental Psychology: Human Perception \& Performance, 16, 766-780.

Fetterman, J. G., Killeen, P. R., \& Hall, S. (1998). Watching the clock. Behavioral Processes, 44, 211-222.

Fraisse, P. (1978). Time and rhythm perception. In E. C. Carterette \& M. P. Friedman (Eds.), Handbook of perception: Vol. VIII. Perceptual coding (pp. 203-254). New York: Academic Press.

GetTy, D. J. (1975). Discrimination of short temporal intervals: A comparison of two models. Perception \& Psychophysics, 18, 1-8.

GibBon, J. (1991). Origins of scalar timing. Learning \& Motivation, 22, 3-38.

GibBon, J. (1992). Ubiquity of scalar timing with a Poisson clock. Journal of Mathematical Psychology, 36, 283-293.

Gibbon, J., Church, R. M., \& Meck, W. H. (1984). Scalar timing in memory. In J. Gibbon \& L. G. Allan (Eds.), Timing and time perception (Annals of the New York Academy of Sciences, Vol. 423, pp. 5277). New York: New York Academy of Sciences.

Grondin, S. (2001). From physical time to the first and second moments of psychological time. Psychological Bulletin, 127, 22-44.

Grondin, S., \& LAFOREST, M. (2004). Discriminating the tempo variations of a musical excerpt. Acoustical Science \& Technology, 25, 159162.

Grondin, S., Meilleur-Wells, G., \& Lachance, R. (1999). When to start explicit counting in time-intervals discrimination task: A critical point in the timing process of humans. Journal of Experimental Psychology: Human Perception \& Performance, 25, 993-1004.

Grondin, S., Ouellet, B., \& Roussel, M.-È. (2004). Benefits and limits of explicit counting for discriminating temporal intervals. $\mathrm{Ca}$ nadian Journal of Experimental Psychology, 58, 1-12.

Halpern, A. R. (1988). Perceived and imagined tempos of familiar songs. Music Perception, 6, 193-202.

Hinton, S. C., Harrington, D. L., Binder, J. R., Durgerian, S., \& Rao, S. M. (2004). Neural systems supporting timing and chronometric counting: An fMRI study. Cognitive Brain Research, 21, 183192.

Hinton, S. C., \& Rao, S. M. (2004). "One-thousand one . . onethousand two ...": Chronometric counting violates the scalar property in interval timing. Psychonomic Bulletin \& Review, 11, 24-30.

Killeen, P. R., \& Weiss, N. A. (1987). Optimal timing and the Weber function. Psychological Review, 94, 455-468.

Kristofferson, A. B. (1980). A quantal step function in duration discrimination. Perception \& Psychophysics, 27, 300-306.

LeJeune, H., \& WeARden, J. H. (2006). Scalar properties in animal tim- 
ing: Conformity and violations. Quarterly Journal of Experimental Psychology, 59, 1875-1908.

Levitin, D. J., \& CooK, P. R. (1996). Memory for musical tempo: Additional evidence that auditory memory is absolute. Perception \& Psychophysics, 58, 927-935.

Macar, F., Grondin, S., \& CASINI, L. (1994). Controlled attention sharing influences time estimation. Memory \& Cognition, 22, 673-686.

McAuley, J. D., \& Jones, M. R. (2003). Modeling effects of rhythmic context on perceived duration: A comparison of interval and entrainment approaches to short-interval timing. Journal of Experimental Psychology: Human Perception \& Performance, 29, 1102-1125.

McAuley, J. D., Jones, M. R., Holub, S., Johnston, H. M., \& Miller, N. S. (2006). The time of our lives: Life span development of timing and event tracking. Journal of Experimental Psychology: General, 135, 348-367.

Petrusic, W. M. (1984). Explicit counting and time-order errors in duration discrimination. In J. Gibbon \& L. G. Allan (Eds.), Timing and time perception (Annals of the New York Academy of Sciences, Vol. 423, pp. 630-633). New York: New York Academy of Sciences.

Petrusic, W. M. (2002). Chronometric mental counting in duration discrimination. In J. A. Da Silva, E. H. Matsushima, \& N. P. Ribeiro-Filho (Eds.), Fechner Day 2002: Proceedings of the 18th Annual Meeting of the International Society for Psychophysics (pp. 116-121). Ribeirao Preto, Brazil: Editora Legis Summa Ltda.

Rakitin, B. C., Stern, Y., \& Malapani, C. (2005). The effects of aging on time reproduction in delayed free-recall. Brain \& Cognition, 58, 17-34.

Rammsayer, T., \& Ulrich, R. (2001). Counting models of temporal discrimination. Psychonomic Bulletin \& Review, 8, 270-277.
Sheldon, D. A. (1994). Effects of tempo, musical experience, and listening modes on tempo modulation perception. Journal of Research in Music Education, 42, 190-202.

WILCOX, R. R. (1998). How many discoveries have been lost by ignoring modern statistical methods? American Psychologist, 53, 300-314.

\section{NOTES}

1. We thank an anonymous reviewer for noting this distinction between singing and counting.

2. It is very demanding for musicians to refrain from beating time, and because of that, we restricted this portion of the experiment to the segmentation conditions. For nonmusicians, there were no significant differences for the sing or count conditions between people who had the no-segmentation condition first and those who did not; fatigue is therefore an unlikely explanation for the difference in CVs between musicians and nonmusicians.

3. A 1-10 loop was used for counting, because the experiment was conducted in French, in which each digit is pronounced as a monosyllable (even quatre, 4). Some higher numbers (e.g., 14, 17, 18, 19, 22), however, are bisyllabic, and some (e.g., 21) are trisyllabic.

4. Only 11 nonmusician participants were included in this analysis because 1 participant wrote only one letter instead of the complete word reached at the end of the trial in the signing condition. Therefore, it was not possible to determine the points reached by this participant in the singing condition.

(Manuscript received August 26, 2008; revision accepted for publication April 25, 2009.) 